\title{
Imaging Single-mRNA Localization and Translation in Live Neurons
}

\author{
Byung Hun Lee ${ }^{1}$, Seong-Woo Bae ${ }^{1}$, Jaeyoun Jay Shim ${ }^{1}$, Sung Young Park ${ }^{2}$, and Hye Yoon Park ${ }^{1,3, *}$
}

\begin{abstract}
Local protein synthesis mediates precise spatio-temporal regulation of gene expression for neuronal functions such as long-term plasticity, axon guidance and regeneration. To reveal the underlying mechanisms of local translation, it is crucial to understand mRNA transport, localization and translation in live neurons. Among various techniques for mRNA analysis, fluorescence microscopy has been widely used as the most direct method to study localization of mRNA. Live-cell imaging of single RNA molecules is particularly advantageous to dissect the highly heterogeneous and dynamic nature of messenger ribonucleoprotein (mRNP) complexes in neurons. Here, we review recent advances in the study of mRNA localization and translation in live neurons using novel techniques for single-RNA imaging.
\end{abstract}

\section{INTRODUCTION}

The localization and subsequent translation of mRNA are important means of regulating gene expression with high spatial and temporal control. In neurons, targeting mRNA to specific sites and synthesizing proteins where and when they are needed is particularly beneficial because neurons have long dendrites and axons extending from a few hundred microns to up to a meter from the cell body. The physiological roles of mRNA localization in neurons have been implicated in various neuronal functions such as synaptic plasticity, axonal guidance and regeneration. However, there are many remaining questions regarding how mRNAs navigate through the complex neuronal arbors, how they are captured in specific regions, and how local translation is regulated (Buxbaum et al., 2015; Holt and Schuman, 2013; Hutten et al., 2014; Xing and Bassell, 2013). Observing the dynamics of mRNA localization and translation will provide critical information to help understand

\footnotetext{
${ }^{1}$ Department of Physics and Astronomy, Seoul National University, Seoul 08826, Korea, ${ }^{2}$ Center for RNA Research, Institute for Basic Science, Seoul 08826, Korea, ${ }^{3}$ The Institute of Molecular Biology and Genetics, Seoul National University, Seoul 08826, Korea

${ }^{*}$ Correspondence: hyeyoon.park@snu.ac.kr
}

Received 16 November, 2016; revised 20 December, 2016; accepted 21 December, 2016; published online 29 December, 2016

Keywords: live-cell imaging, local translation, MS2-GFP, RNA localization, single-molecule imaging how precise spatio-temporal regulation of gene expression is processed.

Most previous studies have used in situ hybridization (ISH) methods (Lawrence and Singer, 1985) to visualize RNA molecules inside a cell. In particular, fluorescence in situ hybridization (FISH) enables the detection of individual RNA molecules with various signal amplification procedures in fixed cells. Single-molecule FISH (smFISH), which uses multiple fluorescent probes hybridized to a single mRNA (Femino et al., 1998; Raj et al., 2008), is widely used to profile transcription, localization and degradation of RNA. However, FISH experiments using fixed cells cannot provide temporal information on RNA regulation. Recent innovations in live-cell imaging technologies have made it possible to observe the sequence of molecular events in real time, which is critical to our understanding of mRNA dynamics (Moon et al., 2016; Spille and Kubitscheck, 2015). By imaging single mRNA molecules in live cells, we can begin to understand the causeand-effect relationship and to model the kinetics of RNA regulation more quantitatively and predictably.

In this review, we provide a brief overview of recent progress in studies on mRNA localization and translation in neurons. In particular, we highlight several recent reports that have used single-molecule imaging techniques to contribute to our knowledge of mRNA translocation in dendrites and axons. Additionally, we review new technical developments for multicolor imaging of single mRNAs and their translational activities. These approaches will provide a powerful toolkit to help understand the molecular mechanisms of RNA localization and local translation in neurons, with unprecedented temporal and spatial resolution.

\section{SINGLE-mRNA TRAFFICKING IN DENDRITES}

Since the discovery of polysomes at the base of dendritic spines (Steward and Levy, 1982), many lines of evidence have indicated that de novo protein synthesis in dendrites is required for long-term synaptic plasticity (Hanus and Schuman, 2013; Jung et al., 2014). Deep RNA sequencing revealed 2,550 mRNAs that are present in dendrites and axons in the hippocampus (Cajigas et al., 2012). A large fraction of these mRNAs encode synaptic proteins such as signaling molecules, scaffolds and receptors. Among these mRNAs, activity-regulated cytoskeleton-associated protein (Arc), the $\alpha$-subunit of calcium/calmodulin-dependent protein kinase II (CaMKIl $\alpha$ ) and $\beta$ actin mRNAs have been extensively studied to unravel their regulation in live neurons. 


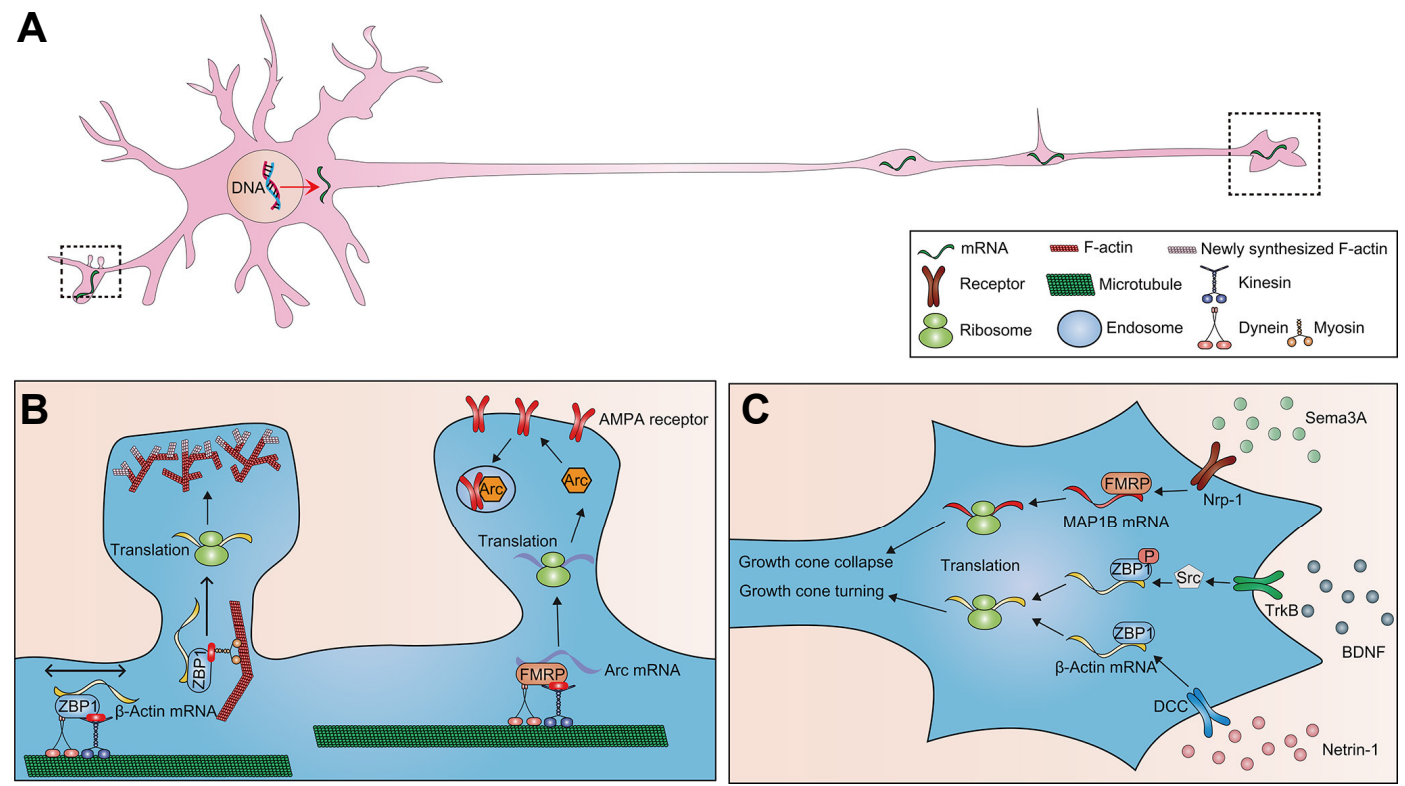

Fig. 1. Schematic diagrams of local translation in dendritic spines and an axonal growth cone. (A) Illustration of mRNA localization in a neuron. (B) Localization of mRNA in dendritic spines. Translation of $\beta$-actin mRNA is repressed by ZBP1 during transport to the localization site. After translation, newly synthesized $\beta$-actin proteins accumulate at the periphery of the spines. Arc mRNA localizes selectively at active synapses and mediates local synthesis of Arc proteins, which play a role in AMPA receptor endocytosis. (C) Localization of mRNA in an axonal growth cone. BDNF and netrin-1 induce local translation of $\beta$-actin mRNA, which mediates growth cone turning toward extracellular cues. Sema3A induces local translation of MAP1B mRNA, which leads to growth cone collapse.

To understand how these mRNAs are sorted and transported to dendrites, it is crucial to track the movement of individual mRNA molecules (Fig. 1A). Single-mRNA tracking has revealed the diverse and stochastic nature of mRNA trafficking (Park et al., 2010). Transport and localization of mRNA depend on the interplay of cis-acting RNA elements, RNA binding proteins (RBPs), and motor proteins (Czaplinski, 2014). Various kinds of RBPs bind to their target mRNAs to form messenger ribonucleoprotein complexes (mRNPs). After recruiting motor proteins, mRNPs are transported along cytoskeletal tracks.

A cis-acting RNA sequence that confers dendritic localization, which is called a dendritic targeting element (DTE), is typically located in the $3^{\prime}$ untranslated region ( $3^{\prime}$ UTR) of the mRNA. Transcripts containing the CaMKIl $\alpha 3^{\prime}$ UTR (Rook et al., 2000) or the Arc 3' UTR (Dynes and Steward, 2007; 2012) exhibit bidirectional transport in dendrites and localize at the base of dendritic spines. A 54-nucleotide-long zipcode in the $\beta$-actin $3^{\prime}$ UTR (Kislauskis et al., 1994) and zipcode binding protein 1 (ZBP1) are required for the localization of $\beta$-actin mRNA in dendritic filopodia and axonal growth cones (Eom et al., 2003; Zhang et al., 2001). Microinjected CaMKIl $\alpha$ and Septin7 mRNAs colocalize in the same particles, while MAP2 mRNAs reside in different particles, suggesting that some RNAs that share a common DTE can be sorted in the same complexes and transported to distal dendrites (Tubing et al., 2010). However, the minimal functional DTE for selective mRNA localization remains largely unknown. For example, there have been conflicting results for defining the DTE in the CaMKIl $\alpha$ 3' UTR (Doyle and Kiebler, 2011).

The cis-acting RNA elements interact with a set of transacting factors or RBPs to form mRNP complexes that are transported along microtubules by kinesin or dynein. Tracking reporter RNAs in Fmr1 knock-out (KO) neurons revealed the important roles of Fragile $X$ mental retardation protein (FMRP) as an adaptor that links mRNA to the kinesin light chain (Dictenberg et al., 2008) and for mRNA delivery to dendritic spines in response to synaptic stimulation (Kao et al., 2010). Live imaging of neurons also showed that Huntingtin ( $\mathrm{Htt}$ ), huntingtin-associated protein 1 (HAP1), KIF5A, and dynein intermediate chain are associated with $\beta$-actin mRNA during dendritic transport (Ma et al., 2011). Knocking down the levels of these proteins reduced the transport of $\beta$-actin mRNA, suggesting that they have roles in mRNA localization in neurons. An actin-based motor, myosin-Va, contributes to the transport of TLS (translocated in liposarcoma)-containing mRNPs in dendritic spines (Yoshimura et al., 2006).

Most previous mRNA tracking studies have been performed using transfection or injection of exogenous reporter mRNAs. However, reporter RNAs usually do not contain all of the cisregulatory sequences and trans-acting RBPs and thus may not represent the true characteristics of endogenous RNAs. To label endogenous mRNA, genetically engineered mice have been generated by inserting MS2 binding site (MBS) stemloops into a target RNA and expressing MS2 capsid protein (MCP)-GFP proteins (Lionnet et al., 2011; Park et al., 2014). Using this technique, all the endogenous $\beta$-actin mRNAs were labeled with the MS2-GFP system in live mice (Park et al., 2014). Tracking the labeled endogenous $\beta$-actin mRNAs revealed that mRNA diffusion was much slower in neurons than in fibroblasts. While most of the $\beta$-actin mRNAs in fibroblasts showed diffusive motion, a small fraction of the $\beta$-actin mRNAs in neurons showed bidirectional motion with a mean speed of $1.3 \mu \mathrm{m} / \mathrm{s}$.

Recently, glutamate uncaging was used to demonstrate that $\beta$-actin mRNAs can localize in the stimulated dendritic region within 15 min of stimulation (Yoon et al., 2016). NMDA recep- 
tors and ZBP1 have important roles in the localization process. In neurons cultured from ZBP1 KO mice, $\beta$-actin mRNAs were transported to the stimulated region; however, they did not maintain their localization. The authors also showed that newly synthesized $\beta$-actin proteins accumulated in the stimulated spine head and that this helped stabilize the expanded spines.

Taken together, tracking single-mRNA movements enables us to reveal how RNA transport and localization happen in live neurons. The current working model is called the "sushi belt model", in which mRNPs stroll along axons and dendrites like sushi plates on a conveyor belt to serve potential customers (Doyle and Kiebler 2011). Those mRNAs may be captured by "synaptic tags" that are relevant to the formation of long-term potentiation (LTP) and long-term depression (LTD) (Frey and Morris, 1997). Further studies using new developments in genetic engineering and in systems biology approaches will elucidate the precise regulatory mechanisms of mRNA localization in dendrites.

\section{IMAGING mRNA LOCALIZATION IN AXONS}

While local translation in dendrites is well accepted, local protein synthesis in axons has been controversial over the years. Although a few early studies reported the presence of ribosomes in axons (Bunge, 1973; Tennyson, 1970; Zelena, 1970), the low density of axonal mRNAs and ribosomes supported the traditional view that axonal proteins are synthesized in the cell body. Later, however, two studies showed evidence for local translation in the axons. Campbell and Holt observed that growth cones of retinal ganglion neurons lose their ability to turn away from a source of Sema3A when translation is inhibited (Campbell and Holt, 2001). Inhibition of protein translation also blocked Sema3A-induced collapse of growth cones. Zhang and Poo showed that localized synaptic potentiation by BDNF requires presynaptic protein translation in Xenopus nerve-muscle cultures (Zhang and Poo, 2002). Since then, there have been many studies on mRNA localization in axons and on the implications of those localized mRNAs for axon guidance, survival and regeneration (Gumy et al., 2014; Jung et al., 2012).

BDNF- or netrin-1-induced growth cone turning is mediated by local translation of $\beta$-actin mRNA (Leung et al., 2006; Yao et al., 2006). BDNF induces Src-mediated ZBP1 phosphorylation, which releases ZBP1 from $\beta$-actin mRNA (Huttelmaier et al., 2005). After ZBP1 is dissociated, $\beta$-actin proteins are locally synthesized from the mRNA and then direct growth cone turning. In a similar mechanism, MAP1B mRNAs are also locally translated in response to Sema3A (Li et al., 2010). It has been suggested that FMRP has a role in protein synthesis-dependent collapse of growth cones induced by Sema3A (Fig. 1C). In addition, branching of sensory axons requires local protein synthesis and the stalling of mitochondria (Spillane et al., 2013). Using a GFP reporter construct containing the $3^{\prime}$ UTR of $\beta$-actin mRNA, the authors found that hot spots of axonal translation occur at the base of branches that also contained mitochondria.

RBPs have important roles in mRNA transport as well as in blocking translation. ZBP1 is colocalized with Myosin-Va in axons, and inhibition of Myosin-Va function increases the number of motile ZBP1 particles (Nalavadi et al., 2012). Furthermore, some RBPs can bind to several species of mRNAs. In dorsal root ganglion neurons, SFPQ, a splicing factor, works as an RBP binding to Lmnb2, Bcl2l2, Impa1 and Creb1 mRNAs (Cosker et al., 2016). SFPQ mediates the coassembly of Lmnb2 and Bcl2I2 mRNAs in RNA granules. In addition, SFPQ plays a role in the axonal transport of these mRNAs to promote axon survival.

In central nervous system (CNS) axons, studies on mRNA localization have been facilitated by the use of microfluidic devices that separate axons from cell bodies and dendrites (Taylor et al., 2005). Using a microfluidic device in conjunction with microarray analysis and FISH, many species of mRNAs have been detected in the axons of CNS neurons as well as of peripheral nervous system (PNS) neurons. Studies of mature cortical axons have identified mRNAs that are involved in the translation machinery, transport, cytoskeletal components and mitochondrial maintenance (Taylor et al., 2009).

To date, there are only a few studies on live-cell imaging of axonal mRNA transport. Using the MS2-GFP system, Bi et al. demonstrated that the $5^{\prime}$ and $3^{\prime}$ UTRs of kor (K-opioid receptor) mRNA are required for its axonal transport in dorsal root ganglion (DRG) neurons (Bi et al., 2006). More recently, a molecular beacon method was used to visualize Nefl (Neurofilament-L) mRNA in axons of primary mouse cortical neurons and human motor neurons derived from iPS cells (Alami et al., 2014). Simultaneous imaging of Nefl mRNA and trans-active response DNA-binding protein 43 (TDP-43), an RBP related to amyotrophic lateral sclerosis (ALS), revealed that TDP-43 promotes anterograde transport of Nefl mRNP granules. This result suggests that TDP-43 has a role in the delivery of target mRNA to distal axons.

With the development of microfluidic devices, RNA labeling techniques, and high-resolution live-cell imaging, it is now more feasible to study mRNA transport in axons. For instance, integrated microfluidic platforms are developed for investigating regeneration of injured axons (Kim et al., 2012). Neurons cultured from MCP $\times$ MBS knock-in mice now allow us to image single endogenous mRNAs without transfection steps (Park et al., 2014). Because local translation plays a crucial role in axon development and regeneration, it is important to understand the molecular mechanisms that govern mRNA trafficking and localization in axons.

\section{MONITORING TRANSLATION FROM A SINGLE mRNA IN LIVE NEURONS}

Translation is a highly regulated, complex biological process in which mRNA is decoded by a ribosome to produce a protein. Several whole-genome techniques for studying translation have been developed based on RNA sequencing (RNA-seq) or mass spectrometry (MS). Ribosome profiling, for example, measures ribosome-protected regions of mRNAs with nextgeneration sequencing (NGS), providing position information on translating ribosomes throughout the cellular transcriptome (Ingolia et al., 2009). Global, averaged measurements of translation initiation, elongation, and termination rates can be made by ribosome profiling (Ingolia et al., 2011). Another sequencingbased technique called translating ribosome affinity purification (TRAP) (Heiman et al., 2008) or Ribotag (Sanz et al., 2009) relies on pulling down GFP- or HA-tagged ribosomal proteins and then the identification of mRNAs associated with them. MS-based methods such as pulsed stable isotope labeling by amino acids in cell culture (SILAC) (Schwanhausser et al., 2009) and biorthogonal noncanonical amino acid tagging (BONCAT) (Dieterich et al., 2006) have been used to identify newly synthesized proteins after adding labeled amino acids.

Because genome-wide methods can only detect the average behavior of many cells, imaging-based techniques have been developed to study the dynamics of translation in single cells (Chao et al., 2012). Fluorescent proteins have been used to 


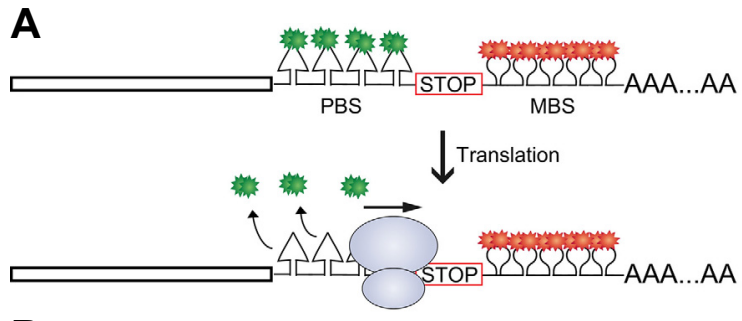

B

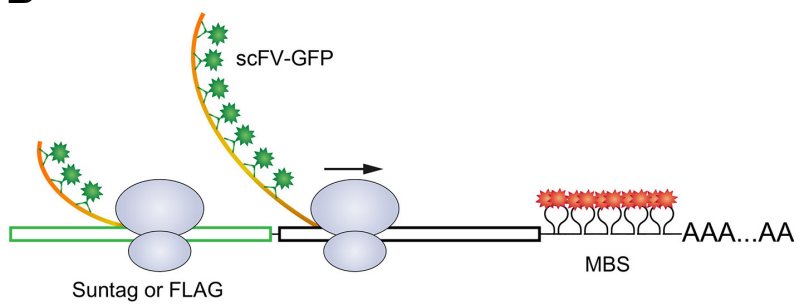

Fig. 2. Schematic diagrams of the TRICK and Suntag (or FLAG)MBS systems. (A) Schematic of the TRICK system. Both PCP-GFP and MCP-RFP are bound to the untranslated mRNA. During translation, ribosomes read through the PBS sequence and knock off PCP-GFP from the mRNA. After the first round of translation, the mRNA is only labeled with RFP. (B) Suntag (or FLAG)-MBS system. Translation of RFP-labeled mRNA can be monitored by the fluorescence signal from scFV-GFP clustered on the nascent polypeptides of Suntag (or FLAG).

detect local protein synthesis (Aakalu et al., 2001); however, the long maturation time of fluorescent proteins has been a major limitation of such methods. As an attempt to visualize translation from single mRNAs, Halstead et al. developed an RNA biosensor termed translating RNA imaging by coat protein knock-off (TRICK) that can distinguish untranslated mRNAs from those that have undergone at least one round of translation (Halstead et al., 2015). In that study, orthogonal bacteriophage PP7 and MS2 stem-loops were used to label mRNA with two different fluorescent proteins (Fig. 2A). Simultaneous expression of the PP7 capsid protein fused with green fluorescent protein (PCP-GFP) and the MS2 capsid protein fused with red fluorescent protein (MCP-RFP) resulted in yellow fluorescent signals from untranslated mRNAs. During the first round of translation, PCP-GFP is removed from the mRNA as a ribosome traverses the coding region that contains the PP7 stemloops. Thus, translated mRNAs are labeled only with MCP-RFP bound to the MS2 stem-loops in the $3^{\prime}$ UTR.

While the TRICK technique provides information on the first round of mRNA translation, recent studies by four independent groups (Morisaki et al., 2016; Wang et al., 2016; Wu et al., 2016; Yan et al., 2016) have demonstrated two-color imaging of mRNA and its nascent polypeptides. These four studies used the MS2 or PP7 systems to label mRNA and the Suntag (Tanenbaum et al., 2014) or FLAG tag (Viswanathan et al., 2015) systems to label nascent proteins. Fluorescence-tagged MS2 or PP7 capsid proteins were expressed in cells, providing a strong fluorescence signal from each mRNA. The Suntag and FLAG tag systems use fluorescently labeled antibody fragments that bind to multiple copies of a short epitope on each polypeptide. An mRNA undergoing active translation can be identified by the colocalization of two fluorescent signals from the mRNA and nascent proteins.
Two of the studies (Wang et al., 2016; Wu et al., 2016) used the Suntag-MS2 technique not only in cell lines but also in hippocampal neurons. They used ornithine decarboxylase (ODC) or auxin-induced degron (AID) to facilitate rapid degradation of the epitope array after translation. This allowed them to distinguish nascent polypeptides translated on the mRNA from the background of freely diffusing polypeptides in the cytosol. Wang et al. found that the Arc $3^{\prime}$ UTR increased the number of translation sites in the dendrites of neurons. Wu et al. showed that $\sim 40 \%$ of the reporter mRNAs were translated in the proximal dendrites; however, only $\sim 10 \%$ were translated in the distal dendrites. Both studies reported that translating polysomes exhibited active transport in the dendrites of neurons. This result contradicts the previous speculation that translation is repressed during the transport of mRNA to localization sites.

Although these findings were obtained using exogenous reporters, the tagging method could be applied to endogenous genes with CRISPR/Cas9 genome-editing techniques (Nelles et al., 2016). If we could track endogenous mRNA while measuring its translational output in response to diverse signaling in live tissues, we would be able to uncover many interesting details regarding the dynamics of gene expression. Further improvements in these imaging techniques (Pichon et al., 2016) will certainly offer new insights into the complex regulation of translation at the single-molecule level in situ.

\section{CONCLUSION}

The elongated morphology of neurons requires directed transport of mRNA and local translation of proteins for efficient gene expression in dendrites and axons. Because local protein synthesis has important roles in neuronal development and synaptic plasticity, it is crucial to understand how specific mRNA molecules are targeted to the activated regions for local translation. Recent advances in live-cell single-RNA imaging techniques have significantly increased our knowledge on the dynamics of RNA in neurons. However, many outstanding questions remain. What are the cis-regulatory RNA sequences for selective transport into dendrites and axons? How are these sequences recognized by RBPs and motor proteins? How are mRNPs delivered and captured at the stimulated synapses? Understanding the molecular mechanisms behind RNA localization, translation and degradation in neurons will provide clearer answers to questions about the physiological relationships between gene expression and brain functions.

\section{ACKNOWLEDGMENTS}

This work was supported by the Seoul National University Research Grant in 2015 and the Basic Science Research Program through the National Research Foundation of Korea (NRF) funded by the Ministry of Science, ICT \& Future Planning (2015R1C1A1A02036674). B.H.L., S.-W.B., and J.J.S. were supported by BK21 Research Fellowships from the Ministry of Education of Korea. S.Y.P. was supported by IBS-R008-D1 of the Institute for Basic Science from the Ministry of Science, ICT, and Future Planning of Korea.

\section{REFERENCES}

Aakalu, G., Smith, W.B., Nguyen, N., Jiang, C., and Schuman, E.M. (2001). Dynamic visualization of local protein synthesis in hippocampal neurons. Neuron 30, 489-502.

Alami, N.H., Smith, R.B., Carrasco, M.A., Williams, L.A., Winborn, C.S., Han, S.S.W., Kiskinis, E., Winborn, B., Freibaum, B.D. Kanagaraj, A., et al. (2014). Axonal transport of TDP-43 mRNA granules is impaired by ALS-causing mutations. Neuron 81, 536- 
543.

Bi, J., Tsai, N.P., Lin, Y.P., Loh, H.H., and Wei, L.N. (2006). Axonal mRNA transport and localized translational regulation of kappaopioid receptor in primary neurons of dorsal root ganglia. Proc. Natl. Acad. Sci. USA 103, 19919-19924.

Bunge, M.B. (1973). Fine structure of nerve fibers and growth cones of isolated sympathetic neurons in culture. J. Cell Biol. 56, 713-735.

Buxbaum, A.R., Yoon, Y.J., Singer, R.H., and Park, H.Y. (2015). Single-molecule insights into mRNA dynamics in neurons. Trends Cell Biol. 25, 468-475.

Cajigas, I.J., Tushev, G., Will, T.J., Dieck, S.T., Fuerst, N., and Schuman, E.M. (2012). The local transcriptome in the synaptic neuropil revealed by deep sequencing and high-resolution imaging. Neuron $74,453-466$.

Campbell, D.S., and Holt, C.E. (2001). Chemotropic responses of retinal growth cones mediated by rapid local protein synthesis and degradation. Neuron 32, 1013-1026.

Chao, J.A., Yoon, Y.J., and Singer, R.H. (2012). Imaging translation in single cells using fluorescent microscopy. Cold Spring Harb Perspect Biol. 4.

Cosker, K.E., Fenstermacher, S.J., Pazyra-Murphy, M.F., Elliott, H.L., and Segal, R.A. (2016). The RNA-binding protein SFPQ orchestrates an RNA regulon to promote axon viability. Nat. Neurosci. 19, 690-696.

Czaplinski, K. (2014). Understanding mRNA trafficking: Are we there yet? Semin. Cell Dev. Biol. 32, 63-70.

Dictenberg, J.B., Swanger, S.A., Antar, L.N., Singer, R.H., and Bassell, G.J. (2008). A direct role for FMRP in activity-dependent dendritic mRNA transport links filopodial-spine morphogenesis to fragile X syndrome. Dev. Cell 14, 926-939.

Dieterich, D.C., Link, A.J., Graumann, J., Tirrell, D.A., and Schuman, E.M. (2006). Selective identification of newly synthesized proteins in mammalian cells using bioorthogonal noncanonical amino acid tagging (BONCAT). Proc. Natl. Acad. Sci. USA 103, 9482-9487.

Doyle, M., and Kiebler, M.A. (2011). Mechanisms of dendritic mRNA transport and its role in synaptic tagging. EMBO J. 30, 3540-3552.

Dynes, J.L., and Steward, O. (2007). Dynamics of bidirectional transport of Arc mRNA in neuronal dendrites. J. Comp. Neurol. 500, 433-447.

Dynes, J.L., and Steward, O. (2012). Arc mRNA docks precisely at the base of individual dendritic spines indicating the existence of a specialized microdomain for synapse-specific mRNA translation. J. Comp. Neurol. 520, 3105-3119.

Eom, T., Antar, L.N., Singer, R.H., and Bassell, G.J. (2003). Localization of a beta-actin messenger ribonucleoprotein complex with zipcode-binding protein modulates the density of dendritic filopodia and filopodial synapses. J. Neurosci. 23, 10433-10444.

Femino, A.M., Fay, F.S., Fogarty, K., and Singer, R.H. (1998). Visualization of single RNA transcripts in situ. Science 280,585 590.

Frey, U., and Morris, R.G.M. (1997). Synaptic tagging and longterm potentiation. Nature 385, 533-536.

Gumy, L.F., Katrukha, E.A., Kapitein, L.C., and Hoogenraad, C.C. (2014). New insights into mRNA trafficking in axons. Dev. Neurobiol. 74, 233-244.

Halstead, J.M., Lionnet, T., Wilbertz, J.H., Wippich, F., Ephrussi, A., Singer, R.H., and Chao, J.A. (2015). An RNA biosensor for imaging the first round of translation from single cells to living animals. Science $347,1367-1371$.

Hanus, C., and Schuman, E.M. (2013). Proteostasis in complex dendrites. Nat. Rev. Neurosci. 14, 638-648.

Heiman, M., Schaefer, A., Gong, S., Peterson, J.D., Day, M., Ramsey, K.E., Suarez-Farinas, M., Schwarz, C., Stephan, D.A., Surmeier, D.J., et al. (2008). A translational profiling approach for the molecular characterization of CNS cell types. Cell 135, 738748.

Holt, C.E., and Schuman, E.M. (2013). The central dogma decentralized: new perspectives on RNA function and local translation in neurons. Neuron $80,648-657$.

Huttelmaier, S., Zenklusen, D., Lederer, M., Dictenberg, J., Lorenz, M., Meng, X.H., Bassell, G.J., Condeelis, J., and Singer, R.H. (2005). Spatial regulation of beta-actin translation by Src- dependent phosphorylation of ZBP1. Nature 438, 512-515.

Hutten, S., Sharangdhar, T., and Kiebler, M. (2014). Unmasking the messenger. RNA Biol. 11, 992-997.

Ingolia, N.T., Ghaemmaghami, S., Newman, J.R.S., and Weissman, J.S. (2009). Genome-wide analysis in vivo of translation with nucleotide resolution using ribosome profiling. Science 324, 218223.

Ingolia, N.T., Lareau, L.F., and Weissman, J.S. (2011). Ribosome profiling of mouse embryonic stem cells reveals the complexity and dynamics of mammalian proteomes. Cell 147, 789-802.

Jung, H.S., Yoon, B.C., and Holt, C.E. (2012). Axonal mRNA localization and local protein synthesis in nervous system assembly, maintenance and repair. Nat. Rev. Neurosci. 13, 308324.

Jung, H., Gkogkas, C.G., Sonenberg, N., and Holt, C.E. (2014). Remote control of gene function by local translation. Cell 157, 2640.

Kao, D.I., Aldridge, G.M., Weiler, I.J., and Greenough, W.T. (2010). Altered mRNA transport, docking, and protein translation in neurons lacking fragile $X$ mental retardation protein. Proc. Natl. Acad. Sci. USA 107, 15601-15606.

Kim, H.J., Park, J.W., Byun, J.H., Vahidi, B., Rhee, S.W., and Jeon, N.L. (2012). Integrated microfluidics platforms for investigating injury and regeneration of CNS axons. Ann. Biomed. Eng. 40, 1268-1276.

Kislauskis, E.H., Zhu, X., and Singer, R.H. (1994). Sequences responsible for intracellular localization of beta-actin messenger RNA also affect cell phenotype. J. Cell Biol. 127, 441-451.

Lawrence, J.B., and Singer, R.H. (1985). Quantitative analysis of in situ hybridization methods for the detection of actin gene expression. Nucleic Acids Res. 13, 1777-1799.

Leung, K.M., van Horck, F.P.G., Lin, A.C., Allison, R., Standart, N., and Holt, C.E. (2006). Asymmetrical beta-actin mRNA translation in growth cones mediates attractive turning to netrin-1. Nat. Neurosci. 9, 1247-1256.

Li, C., Bassell, G., and Sasaki, Y. (2010). Fragile X mental retardation protein is involved in protein synthesis-dependent collapse of growth cones induced by Semaphorin-3A. J. Pharmacol. Sci. 112, 241p-241p.

Lionnet, T., Czaplinski, K., Darzacq, X., Shav-Tal, Y., Wells, A.L., Chao, J.A., Park, H.Y., de Turris, V., Lopez-Jones, M., and Singer, R.H. (2011). A transgenic mouse for in vivo detection of endogenous labeled mRNA. Nat. Methods 8, 165-U196.

Ma, B., Savas, J.N., Yu, M.S., Culver, B.P., Chao, M.V., and Tanese, N. (2011). Huntingtin mediates dendritic transport of beta-actin mRNA in rat neurons. Sci. Rep. 1, 140.

Moon, H.C., Lee, B.H., Lim, K., Son, J.S., Song, M.S., and Park, H.Y. (2016). Tracking single mRNA molecules in live cells. J. Phys. D Appl. Phys. 49.

Morisaki, T., Lyon, K., DeLuca, K.F., DeLuca, J.G., English, B.P., Zhang, Z.J., Lavis, L.D., Grimm, J.B., Viswanathan, S., Looger L.L., et al. (2016). Real-time quantification of single RNA translation dynamics in living cells. Science 352, 1425-1429.

Nalavadi, V.C., Griffin, L.E., Picard-Fraser, P., Swanson, A.M., Takumi, T., and Bassell, G.J. (2012). Regulation of zipcode binding protein 1 transport dynamics in axons by myosin Va. J. Neurosci. 32, 15133-15141.

Nelles, D.A., Fang, M.Y., O'Connell, M.R., Xu, J.L., Markmiller, S.J., Doudna, J.A., and Yeo, G.W. (2016). Programmable RNA tracking in live cells with CRISPR/Cas9. Cell 165, 488-496.

Park, H.Y., Buxbaum, A.R., and Singer, R.H. (2010). Single mRNA tracking in live cells. Methods Enzymol. 472, 387-406.

Park, H.Y., Lim, H., Yoon, Y.J., Follenzi, A., Nwokafor, C., LopezJones, M., Meng, X.H., and Singer, R.H. (2014). Visualization of dynamics of single endogenous mRNA labeled in live mouse. Science 343, 422-424.

Pichon, X., Bastide, A., Safieddine, A., Chouaib, R., Samacoits, A., Basyuk, E., Peter, M., Mueller, F., and Bertrand, E. (2016). Visualization of single endogenous polysomes reveals the dynamics of translation in live human cells. J. Cell Biol. 214, 769781.

Raj, A., van den Bogaard, P., Rifkin, S.A., van Oudenaarden, A., and Tyagi, S. (2008). Imaging individual mRNA molecules using multiple singly labeled probes. Nat. Methods 5, 877-879.

Rook, M.S., Lu, M., and Kosik, K.S. (2000). CaMKIlalpha $3^{\prime}$ untranslated region-directed mRNA translocation in living 
neurons: visualization by GFP linkage. J. Neurosci. 20, 63856393.

Sanz, E., Yang, L., Su, T., Morris, D.R., McKnight, G.S., and Amieux, P.S. (2009). Cell-type-specific isolation of ribosomeassociated mRNA from complex tissues. Proc. Natl. Acad. Sci. USA 106, 13939-13944.

Schwanhausser, B., Gossen, M., Dittmar, G., and Selbach, M. (2009). Global analysis of cellular protein translation by pulsed SILAC. Proteomics 9, 205-209.

Spillane, M., Ketschek, A., Merianda, T.T., Twiss, J.L., and Gallo, G. (2013). Mitochondria coordinate sites of axon branching through localized intra-axonal protein synthesis. Cell Rep. 5, 1564-1575.

Spille, J.H., and Kubitscheck, U. (2015). Labelling and imaging of single endogenous messenger RNA particles in vivo. J. Cell Sci. 128, 3695-3706.

Steward, O., and Levy, W.B. (1982). Preferential localization of polyribosomes under the base of dendritic spines in granule cells of the dentate gyrus. J. Neurosci. 2, 284-291.

Tanenbaum, M.E., Gilbert, L.A., Qi, L.S., Weissman, J.S., and Vale, R.D. (2014). A protein-Tagging system for signal amplification in gene expression and fluorescence imaging. Cell 159, 635-646.

Taylor, A.M., Blurton-Jones, M., Rhee, S.W., Cribbs, D.H., Cotman, C.W., and Jeon, N.L. (2005). A microfluidic culture platform for CNS axonal injury, regeneration and transport. Nat. Methods 2, 599-605.

Taylor, A.M., Berchtold, N.C., Perreau, V.M., Tu, C.H., Jeon, N.L., and Cotman, C.W. (2009). Axonal mRNA in uninjured and regenerating cortical mammalian axons. J. Neurosci. 29, 46974707.

Tennyson, V.M. (1970). The fine structure of the axon and growth cone of the dorsal root neuroblast of the rabbit embryo. J. Cell Biol. 44, 62-79.

Tubing, F., Vendra, G., Mikl, M., Macchi, P., Thomas, S., and Kiebler, M.A. (2010). Dendritically localized transcripts are sorted into distinct ribonucleoprotein particles that display fast directional motility along dendrites of hippocampal neurons. J. Neurosci. 30, 4160-4170.
Viswanathan, S., Williams, M.E., Bloss, E.B., Stasevich, T.J., Speer C.M., Nern, A., Pfeiffer, B.D., Hooks, B.M., Li, W.P., English, B.P., et al. (2015). High-performance probes for light and electron microscopy. Nat. Methods 12, 568-576.

Wang, C., Han, B.R., Zhou, R.B., and Zhuang, X.W. (2016). Realtime imaging of translation on single mRNA transcripts in live cells. Cell 165, 990-1001.

Wu, B., Eliscovich, C., Yoon, Y.J., and Singer, R.H. (2016). Translation dynamics of single mRNAs in live cells and neurons. Science 352, 1430-1435.

Xing, L., and Bassell, G.J. (2013). mRNA localization: an orchestration of assembly, traffic and synthesis. Traffic 14, 2-14.

Yan, X.W., Hoek, T.A., Vale, R.D., and Tanenbaum, M.E. (2016). Dynamics of translation of single mRNA molecules in vivo. Cell 165, 976-989.

Yao, J.Q., Sasaki, Y., Wen, Z.X., Bassell, G.J., and Zheng, J.Q. (2006). An essential role for beta-actin mRNA localization and translation in $\mathrm{Ca}^{2+}$-dependent growth cone guidance. Nat. Neurosci. 9, 1265-1273.

Yoon, Y.J., Wu, B., Buxbaum, A.R., Das, S., Tsai, A., English, B.P., Grimm, J.B., Lavis, L.D., and Singer, R.H. (2016). Glutamateinduced RNA localization and translation in neurons. Proc. Natl. Acad. Sci. USA 113, E6877-E6886.

Yoshimura, A., Fujii, R., Watanabe, Y., Okabe, S., Fukui, K., and Takumi, T. (2006). Myosin-Va facilitates the accumulation of mRNA/protein complex in dendritic spines. Curr. Biol. 16, 23452351.

Zelena, J. (1970). Ribosome-like particles in myelinated axons of the rat. Brain Res. 24, 359-363.

Zhang, X.H., and Poo, M.M. (2002). Localized synaptic potentiation by BDNF requires local protein synthesis in the developing axon. Neuron 36, 675-688.

Zhang, H.L., Eom, T., Oleynikov, Y., Shenoy, S.M., Liebelt, D.A., Dictenberg, J.B., Singer, R.H., and Bassell, G.J. (2001). Neurotrophin-induced transport of a beta-actin mRNP complex increases beta-actin levels and stimulates growth cone motility. Neuron 31, 261-275. 